\title{
Anticancer Effects of a New Aminosugar-conjugated Platinum Complex Agent Against Cisplatin-resistant Gastric Cancer
}

\author{
NORIYUKI HAYASHI ${ }^{1}$, HIROMI KATAOKA ${ }^{1}$, SHIGENOBU YANO ${ }^{2,3}$, JUN-ICHI KIKUCHI ${ }^{2}$, \\ MAMORU TANAKA ${ }^{1}$, HIROTADA NISHIE ${ }^{1}$, YUMA KINOSHITA ${ }^{4}$, MIKI HATANO ${ }^{4}$, \\ AKIHIRO NOMOTO ${ }^{4}$, AKIYA OGAWA ${ }^{4}$, MASAHIRO INOUE ${ }^{5}$, TSUTOMU MIZOSHITA ${ }^{1}$, \\ TAKAYA SHIMURA ${ }^{1}$, YOSHINORI MORI $^{1}$, EIJI KUBOTA ${ }^{1}$, SATOSHI TANIDA $^{1}$ and TAKASHI JOH ${ }^{1}$ \\ ${ }^{1}$ Department of Gastroenterology and Metabolism, \\ Nagoya City University Graduate School of Medical Sciences, Nagoya, Japan; \\ ${ }^{2}$ Graduate School of Materials Science, Nara Institute of Science and Technology, Nara, Japan; \\ ${ }^{3}$ Office of Society-Academia Collaboration for Innovation, Kyoto University, Kyoto, Japan; \\ ${ }^{4}$ Department of Applied Chemistry, Graduate School of Engineering, Osaka Prefecture University, Osaka, Japan; \\ ${ }^{5}$ Department of Biochemistry, Osaka Medical Centre for Cancer and Cardiovascular Diseases, Osaka, Japan
}

\begin{abstract}
Background/Aim: Resistance against cisplatin is a problem for the success of gastric cancer chemotherapy. Herein, we evaluated the antitumor effect of a new aminosugar-conjugated, mono-functional platinum complex (Pt-Oqn), which forms a single covalent bond with DNA. Materials and Methods: We compared the cytotoxicity of PtOqn to that of cisplatin (CDDP), oxaliplatin $(L-O H P)$ and carboplatin (CBDCA). We also compared Pt-Oqn and cisplatin for DNA double-strand breaks based on phosphorylated histone $H 2 A X$ levels in cancer cells and antitumor effects in xenograft models. Results: The resistance factor (RF) for Pt-Oqn was low among the four drugs, indicating the potential of Pt-Oqn for overcoming CDDPinduced resistance. In MKN45-R cells, $\gamma-H 2 A X$ protein increased following treatment with Pt-Oqn, but not with cisplatin. Finally, Pt-Oqn, but not cisplatin, showed significant antitumor effects in MKN45-R xenografts. Conclusion: This new aminosugar-conjugated platinum complex is a promising candidate agent for overcoming the drug resistance of cisplatin-resistant stomach cancer.
\end{abstract}

This article is freely accessible online.

Correspondence to: Hiromi Kataoka, MD, Ph.D., Department of Gastroenterology and Metabolism, Nagoya City University Graduate School of Medical Sciences, 1 Kawasumi, Mizuho-cho, Mizuho-ku, Nagoya 467-8601, Japan. Tel: +81 528538211, Fax: +81 528520952, e-mail: hkataoka@med.nagoya-cu.ac.jp

Key Words: Platinum complex, cisplatin, gastric cancer, chemotherapy, cisplatin resistance.
The platinum drug cisplatin (CDDP) is used in cancer chemotherapy to treat a variety of tumors (1). Unfortunately, the effectiveness of CDDP treatment is limited by side-effects, such as nephrotoxicity, neurotoxicity, ototoxicity and severe emesis, as well as by the acquisition of drug resistance to cancer chemotherapy, especially in gastric cancer (2). New agents effective against cisplatin-resistant tumors are, thus, a priority in cancer treatment (3) and various platinum complexes have been studied in recent years to overcome these problems (4-7).

CDDP typically binds to and damages genomic DNA through the formation bifunctional intra-strand lesions that inhibit transcription and induce cell death by apoptosis. This mechanism inherently brings with it the above-described sideeffects and tolerance; thus, new platinum drugs would ideally act via different cytotoxic mechanisms from that of CDDP (8).

Recently, numerous monofunctional platinum complexes were developed as novel non-classical anticancer agents (810). These drugs act by forming a single covalent bond with DNA to induce cancer cell death. In this study, we synthesized a new such platinum complex (Pt-Oqn $=[$ chloro $\{\mathrm{N}-(8$-hydroxoquinoline-2-ylmethylidene)- $\beta$-D-glucosamine $\}$ platinum(II)]) as a novel monofunctional platinum anticancer agent and evaluated its cytotoxicity and ability to induce DNA doublestrand breaks in CDDP-sensitive and -resistant gastric cancer cell lines in vitro and in vivo compared to CDDP, carboplatin (CBDCA) and oxaliplatin (L-OHP).

\section{Materials and Methods}

Drugs. CDDP and CBDCA were purchased from Bristol-Myers Co. (Tokyo, Japan). L-OHP was purchased from Yakult Co. (Tokyo, Japan). Pt-Oqn, the newly obtained Schiff base (L) formed from the reaction between $D(+)$-glucosamine and 8-hydroxyquinoline-2- 
carbaldehyde reacted with $\mathrm{Pt}(\mathrm{DMSO})_{2} \mathrm{Cl}_{2}$.to yield $\mathrm{Pt}-\mathrm{Oqn} \bullet 0.5$ $\mathrm{HCl} \bullet 0.5 \mathrm{MeOH}$. Anal. Calcd for Pt-Oqn $0.5 \mathrm{HCl} \bullet 0.5 \mathrm{MeOH}$ : C, 33.13; H, 3.29; N, 4.68. found: C, 32.92; H, 3.24; N, 4.96. ESI-MS $\mathrm{m} / \mathrm{z}:[\mathrm{M}+\mathrm{Na}]^{+}$calcd for $\mathrm{C} 16 \mathrm{H} 17 \mathrm{ClN} 2 \mathrm{NaO} 6 \mathrm{Pt}: 586.03206$, found: 586.03278 .

Measurements. ${ }^{1} \mathrm{H}$ and ${ }^{13} \mathrm{C}-\mathrm{NMR}$ spectra were recorded on JEOL ECX 400 or ECS 400 (400 MHz) spectrometers (JEOL Ltd., Tokyo, Japan). Elemental analysis was carried out on a YANACO MT-5 (Yanaco Analytical Instruments Inc., Kyoto, Japan) and ESI-mass spectra were measured on a JEOL JMS-T100LC (JEOL Ltd.).

Crystallography. Crystallographic data for Pt-Oqn $\bullet 0.5 \mathrm{HCl} \bullet 0.5$ $\mathrm{MeOH}$ was deposited with the Cambridge Crystallographic Data Center (CCDC 1454737): 12 Union Road, Cambridge CB21EZ, UK (Fax: +44 1223336033, e-mail: deposit@ccdc.cam.ac.uk).

Cell culture. The human gastric cancer cell lines MKN28 (Japanese Collection of Research Bioresources, Osaka, Japan) and MKN45 (RIKEN BRC, Ibaragi, Japan) were cultured in RPMI1640 (SigmaAldrich, St. Louis, MO, USA) supplemented with $10 \%$ fetal bovine serum. Cell authentication (STR profile) was performed for all human cancer cell lines (MKN28 and MKN45) by Japanese Collection of Research Bioresources (JCRB) cell bank on February 25, 2014.

Establishment of CDDP-resistant sublines from MKN28 and MKN45. CDDP-resistant MKN28 (MKN28-R) and MKN45 (MKN45-R) cell lines were established by continuous exposure to CDDP starting at $0.5 \mu \mathrm{mol} / 1$ and increasing in a stepwise manner to $10 \mu \mathrm{mol} / \mathrm{l}$ for more than 5 months. Experiments with these sublines were performed after maintenance in CDDP-free medium for 2-3 weeks.

In vitro cytotoxicity. Cell viability was measured by using a WST-8 cell proliferation assay (Dojindo, Kumamoto, Japan). Gastric cancer cells were seeded into 96 -well culture plates at $5 \times 10^{3}$ cells/100 $\mu \mathrm{l} /$ well and incubated overnight. Cells were treated for $48 \mathrm{~h}$ with graded concentrations of CDDP, Pt-Oqn, L-OHP or CBDCA. To determine cell survival, cells were incubated with cell counting kit- 8 solution for $4 \mathrm{~h}$ and absorption at $450 \mathrm{~nm}$ was measured with a microplate reader (SPECTRA MAX340; Molecular Devices, Sunnyvale, CA USA). Cell viability was expressed as a percentage of treated cells $v s$. untreated control cells. The half maximal $(50 \%)$ inhibitory concentration $\left(\mathrm{IC}_{50}\right)$ was calculated. Resistance factor (RF) is defined as the relative ratio of $\mathrm{IC}_{50}$ values in both cell lines (MKN28-R/MKN28 or MKN45-R/MKN45).

Assessment of DNA double-strand breaks. Gastric cancer cells were seeded and incubated overnight. Cells were treated for $48 \mathrm{~h}$ with $\operatorname{CDDP}(25,100,400 \mu \mathrm{mol} / \mathrm{l})$ and Pt-Oqn $(25,100,400 \mu \mathrm{mol} / \mathrm{l})$. Western blotting was performed by using anti- $\gamma \mathrm{H} 2 \mathrm{AX}(1: 2,000$; Bethyl Laboratories, Inc., TX, USA).

Animals and tumor models. Pathogen-free female nude mice (BALB/c Slc-nu/nu) aged 4 weeks and weighing 20-25 g were obtained from Japan SLC (Kyoto, Japan). Animals were allowed to acclimatize for 2 weeks in the animal facility before any interventions were initiated. Xenograft tumor models were established by implanting $1 \times 10^{6}$ gastric cancer cells (MKN45, MKN45-R) subcutaneously under their right flank. The procedures and experiments were approved by Nagoya City University Center for
Experimental Animal Science and mice were cared for according to the guidelines of the Nagoya City University for Animal Experiments.

In vivo treatment. When transplanted tumors grew to approximately $100 \mathrm{~mm}^{3}$, mice were given intraperitoneal injections of CDDP and Pt-Oqn at a dose of $40 \mu \mathrm{mol} / \mathrm{kg}$ on day 1 and 8 . Tumor growth was monitored by measuring tumor volume with Vernier calipers and calculated using the following formula: (length $\times$ width $\times$ width $/ 2$ ). Relative tumor growth was assessed by comparing tumor volume with that measured on day 1 . The Pt-Oqn, CDDP and control groups consisted of 6,5 and 5 mice, respectively. Results were analyzed by multiple testing (Tukey-Kramer method) between groups.

Statistical analysis. The statistical significance of differences was determined using Student's $t$-test or the Tukey-Kramer method. Differences were considered statistically significant at $p<0.05$. Data are expressed as means $\pm \mathrm{SE}$.

\section{Results}

Structure of [Pt-Oqn]. A platinum(II) is coordinated in a square planar geometry composed of a tridentate Schiff base anion $\left(\mathrm{L}^{-}\right)$and a chloride anion (Figure 1). The glucosamine ring takes the usual $\beta-{ }^{4} \mathrm{C}_{1}$ conformation as shown in the ${ }^{1} \mathrm{H}$ NMR study on Pt-Oqn in DMF- $\mathrm{d}_{7} / \mathrm{D}_{2} \mathrm{O}$ (SI).

Pt-Oqn revealed minimal resistance in CDDP-resistant gastric cancer cells. First, we evaluated the cytotoxicity of CDDP, PtOqn, L-OHP and CBDCA against gastric cancer cells. As shown in Table I, Pt-Oqn exhibited the highest cytotoxicity among the four drugs in MKN28-R cells, while, in the MKN45-R cell line, Pt-Oqn exhibited higher cytotoxicity than CDDP and CBDCA. The RF (MKN28-R/MKN28) of Pt-Oqn was also the lowest among the four drugs, indicating that PtOqn most successfully overcame any cross-resistance of CDDP in the MKN28 cells. For RF (MKN45-R/MKN45), PtOqn was 2nd-lowest among the four drugs (CBDCA: 0. 89, Pt-Oqn: 0. 92) with a big difference over the RFs of CDDP (5. 31) and L-OHP (6. 64). These findings together suggested the strong potential of Pt-Oqn for overcoming CDDP-induced resistance in gastric cancer cell lines.

Pt-Oqn induced DNA double-strand breaks in CDDP-resistant gastric cancer cells. Phosphorylated histone H2AX $(\gamma-\mathrm{H} 2 \mathrm{AX})$ was used herein as a marker of DNA damage and, in MKN45 cells treated with either $400 \mu \mathrm{mol} / \mathrm{l}$ CDDP or Pt-Oqn, $\gamma$-H2AX protein levels were increased comparably. On the other hand, in MKN45-R, $\gamma$-H2AX protein levels were increased by PtOqn, but not CDDP, at $400 \mu \mathrm{mol} / \mathrm{l}$ (Figure 2). These results indicate that Pt-Oqn, but not CDDP, induced DNA doublestrand breaks in CDDP-resistant gastric cancer cells.

Pt-Oqn significantly suppressed CDDP-resistant gastric cancer cell growth. Finally, we examined the antitumor effects of CDDP and Pt-Oqn on xenograft tumor models. As shown 

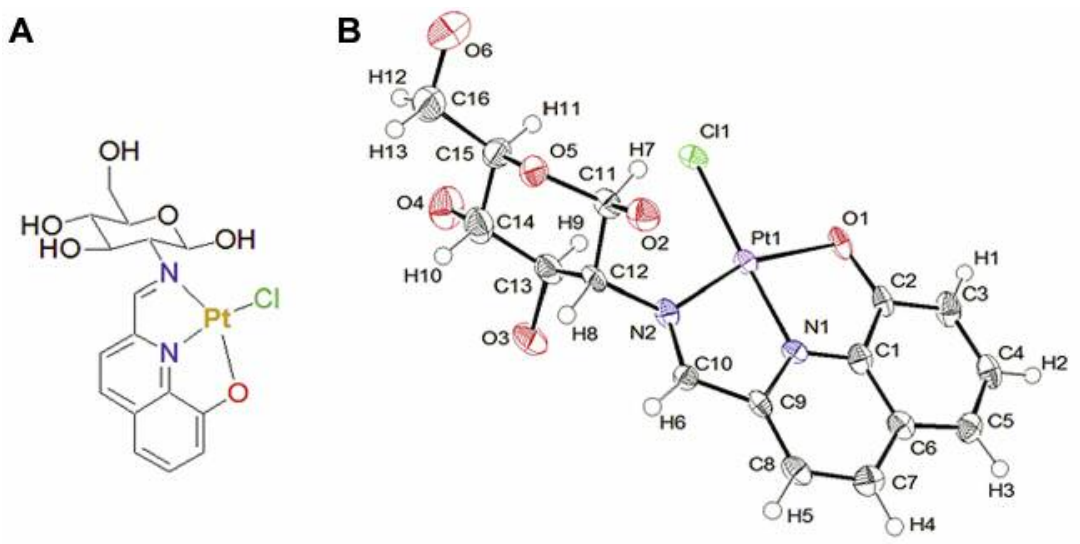

Figure 1. Chemical structure of Pt-Oqn. Structures of the complexes used in this study. (a) Molecular structure of Pt-Oqn. (b) Perspective drawing of Pt-Oqn with atomic numbering scheme in the crystal. Selected bond length (A) and angles (o), Pt1-Cl1 2.3176(17), Pt1-N1 1.1919(6), Pt1-N2 2.036(5), Pt1-O1 2.066(4), C12-N2 1.487(9), Cl1-Pt1-N1 176.24(15), Cl1-Pt1-N2 105.08(15), N1-Pt1- N2 78.4(2), N1-Pt1-O1 82.52(19), Cl1-Pt1O1 94.03(12), N2-Pt1-O1 160.89(19).

in Figure 3, CDDP and Pt-Oqn significantly suppressed tumor growth compared to an untreated control in MKN45 xenografts. On the other hand, only Pt-Oqn significantly suppressed tumor growth compared to controls in the MKN45-R xenografts. None of the therapies had any obvious side-effects, such as diarrhea or weight loss.

\section{Discussion}

CDDP is the prototype member of a group of platinum-based antineoplastic agents that also includes CBDCA (11), with LOHP a new diaminocyclohexane-platinum analogue (12). All of these agents typically form bifunctional intrastrand lesions in cancer cells that bind to and damage genomic doublestranded DNA, thereby inhibiting DNA transcription and blocking the cell cycle to ultimately induce cell death (4).

On the other hand, the recently developed Pt-Oqn is a monofunctional platinum-based complex that tends to form 1,4-GG rather than the 1,2-GG intra-strand crosslinks formed by CDDP (13). As shown in Table I, Pt-Oqn synthesized in this study sustained its cytotoxicity against CDDP-resistant MNK28 and MKN45 cells, while L-OHP and CBDCA had no such effect. We speculate that the superior efficacy of Pt-Oqn against the drug-resistant cancer cell lines lies in the different mechanisms of DNA interaction between monofunctional PtOqn and bifunctional CDDP, CBDCA and L-OHP. Indeed, studies into the acquisition of CDDP resistance by cancer cells, decreased CDDP accumulation, increased CDDP inactivation, whereas abnormalities in apoptotic signaling have proposed several different mechanisms $(14,15)$. Abnormal expression of the adenosine triphosphate (ATP)-binding cassette $(\mathrm{ABC})$ superfamily of transport proteins, multidrug resistance gene-1 $(M D R-1)$ and multidrug resistance-
Table I. In vitro cytotoxicity assay in cisplatin (CDDP)-sensitive and -resistant gastric and colon cancer cell lines.

\begin{tabular}{lccc}
\hline & \multicolumn{3}{c}{$\mathrm{IC}_{50}(\mu \mathrm{M})$} \\
\cline { 2 - 4 } & MKN28 & MKN28-R & Resistance factor \\
\hline Pt-Oqn & $239.4 \pm 12.5$ & $149.8 \pm 23.2$ & 0.63 \\
CDDP & $186.8 \pm 12.8$ & $522.8 \pm 59.1$ & 2.80 \\
L-OHP & $99.1 \pm 13.0$ & $191.7 \pm 26.6$ & 1.93 \\
CBDCA & $123.1 \pm 14.8$ & $422.4 \pm 67.4$ & 3.43 \\
\hline & & $\mathrm{IC}_{50}(\mu \mathrm{M})$ & \\
& & $\mathrm{MKN}^{2}$ & \\
& $\mathrm{MKN}-\mathrm{R}$ & \multirow{2}{*}{ Resistance factor } \\
\hline Pt-Oqn & $187.3 \pm 16.1$ & $171.8 \pm 33.5$ & 0.92 \\
CDDP & $89.9 \pm 22.5$ & $477.4 \pm 47.4$ & 5.31 \\
L-OHP & $13.5 \pm 3.6$ & $89.6 \pm 9.2$ & 6.64 \\
CBDCA & $291.7 \pm 826$ & $258.6 \pm 20.9$ & 0.89 \\
\hline
\end{tabular}

Gastric cancer cells were treated for $48 \mathrm{~h}$ with graded concentrations of CDDP, aminosugar-conjugated mono-functional platinum complex (PtOqn), oxaliplatin (L-OHP) or carboplatin (CBDCA). Cell viability was determined by WST-8 assay and expressed by the half maximal inhibitory concentration $\left(\mathrm{IC}_{50}\right)$. Resistance factor $(\mathrm{RF})$ is defined as the relative ratio of $\mathrm{IC}_{50}$ values in both cell lines (MKN28-R/MKN28 or MKN45-R/MKN45). Data are means of five independent experiments.

associated proteins (MRPs) have been associated with CDDP resistance $(16,17)$. In addition, our previous study showed $A B C B 1$ and $C D K N 2 A$ gene up-regulation in the CDDPresistant gastric cell line MKN45 (18). $A B C B 1$ gene is the most extensively studied ABC superfamily transporter (19), being implicated in MDR involving CDDP accumulation due to active efflux of drugs $(20,21)$. CDKN $2 A$ might also 


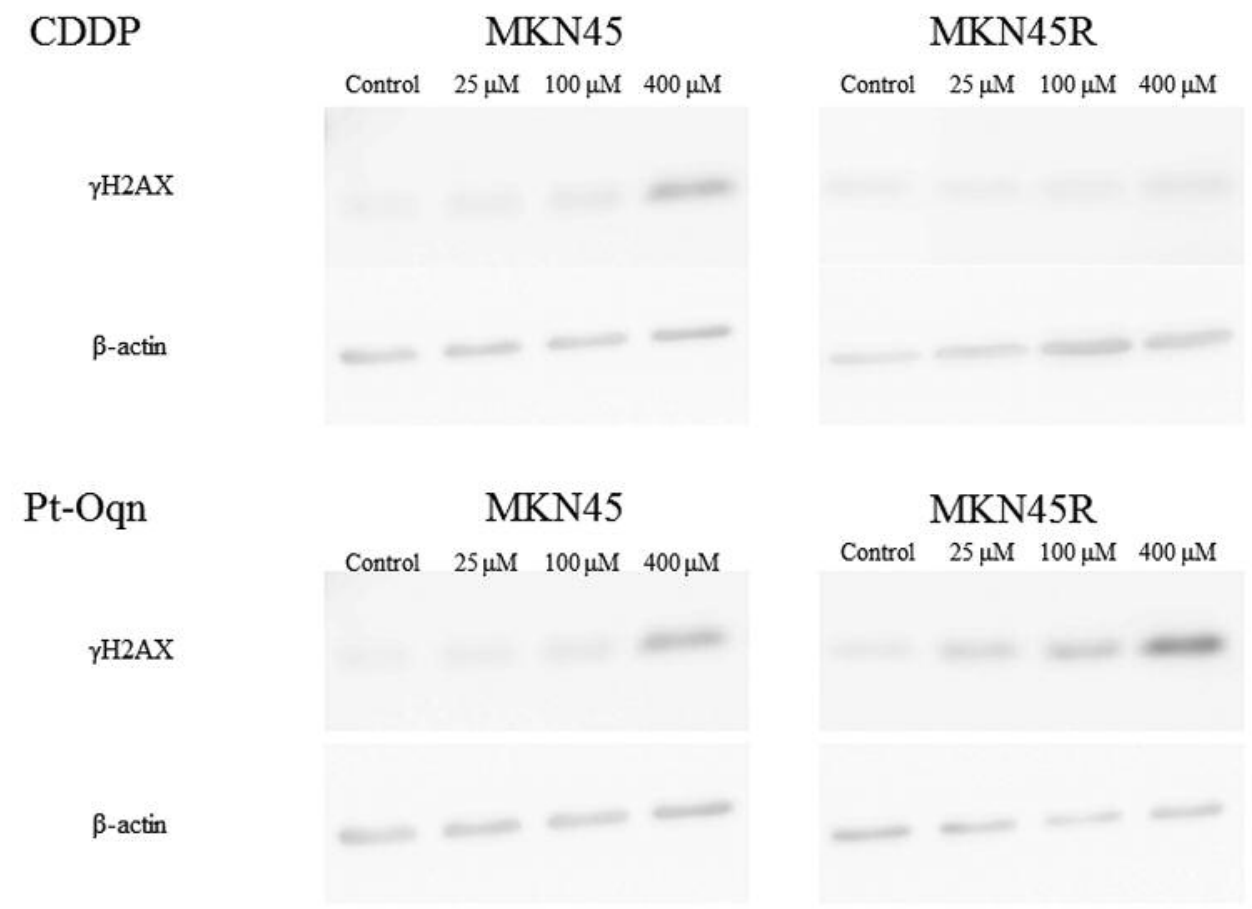

Figure 2. Western blotting analysis of phosphorylated histone H2AX levels in cisplatin (CDDP)-sensitive and -resistant gastric and colon cancer cell lines. Western blots of cell lysates performed at $48 \mathrm{~h}$ after treatment with Pt-Oqn or CDDP were labeled with an antibody against $\gamma H 2 A X$, which detects double-strand breaks. Pt-Oqn, but not CDDP, induced DNA double-strand breaks in the CDDP-resistant gastric cancer cells at $400 \mu$ mol/l.
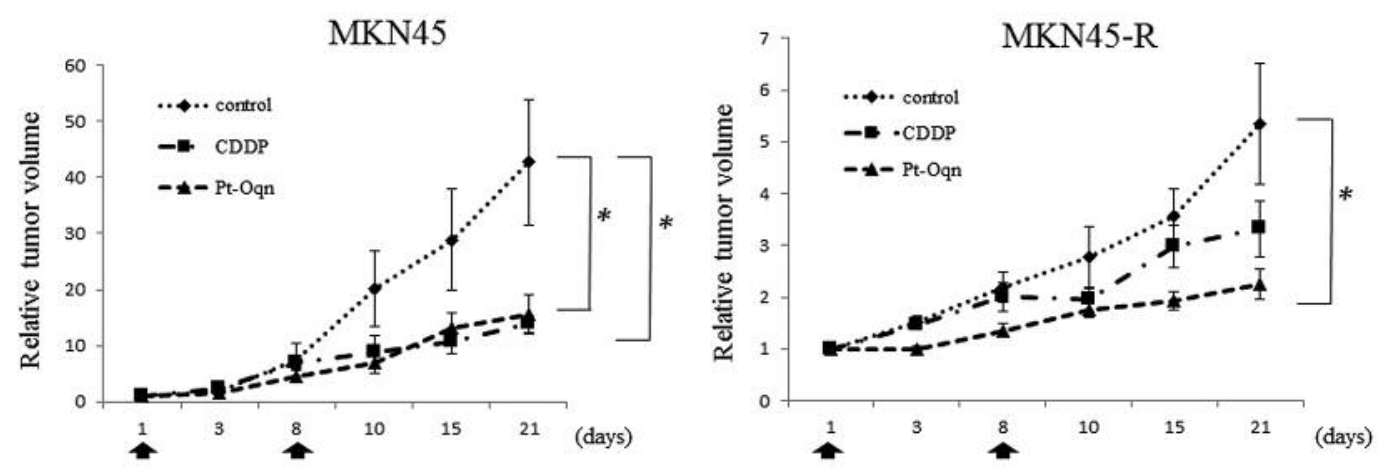

Figure 3. Pt-Oqn significantly suppressed cisplatin (CDDP)-resistant gastric cancer cell proliferation in a xenograft model. Xenograft tumor models were established by implanting $1 \times 10^{6}$ gastric cancer cells $(M K N 45, M K N 45-R)$ subcutaneously under the right flank of mice. Mice were given an intraperitoneal injection of CDDP or Pt-Oqn at a dose of $40 \mu \mathrm{mol} / \mathrm{kg}$. Injection was performed on day 1 and day 8 and tumor volumes were monitored for 21 days in total. Data are means $\pm S E$ ( $n=5$ for control and CDDP $n=5$ for Pt-Oqn). * $p<0.05$.

enhance CDDP resistance by prolonging the cell cycle at the $\mathrm{G}_{1}$-S transition, which allows for DNA repair (22).

Phosphorylation of histone $\mathrm{H} 2 \mathrm{AX}(\gamma \mathrm{H} 2 \mathrm{AX})$ is another protein that forms part of the DNA repair complex in response to double-strand breaks, also used as a marker of DNA damage signal by ionizing radiation and several anticancer reagents (23-25). The mechanism of $\gamma \mathrm{H} 2 \mathrm{AX}$ formation by CDDP treatment involves replication fork collapse and subsequent DNA double-strand break formation at sites of interstrand crosslinking immediately after DNA double-strand breaks $(26,27)$.In our study, Pt-Oqn induced DNA doublestrand breaks in CDDP-resistant gastric cancer cells that CDDP could not at the same concentrations (Figure 2).

In conclusion, a newly synthesized monofunctional $\mathrm{Pt}$ complex that binds to DNA in a monodentate fashion, Pt-Oqn, shows strong activity against CDDP-resistant gastric cancer 
cells and is considered a promising candidate for the nextgeneration platinum-based antineoplastic agents.

\section{Grant Support}

This work was supported by JSPS KAKENHI grant numbers 19350031, 23590923, 25288028, 26288037, 26410056 and 26620154

\section{Conflicts of Interest}

The Authors state that they have no potential conflicts of interest to disclose.

\section{Acknowledgements}

The Authors are grateful to Yukimi Ito at Nagoya City University Graduate School of Medical Sciences for technical assistance and the Core Laboratory, Nagoya City University Graduate School of Medical Sciences, for the use of laboratory equipment.

\section{References}

1 Mori R, Yoshida K, Tanahashi T, Yawata K, Kato J, Okumura N, Tsutani Y, Okada M, Oue N and Yasui W: Decreased FANCJ caused by $5 \mathrm{FU}$ contributes to the increased sensitivity to oxaliplatin in gastric cancer cells. Gastric Cancer 16: 345-354, 2013.

2 Liu Z, Liu R, Zhou Z, Zu Y and Xu F: Structural changes of linear DNA molecules induced by cisplatin. Biochem Biophys Res Commun 457: 688-692, 2015.

3 Jung Y and Lippard SJ: Direct cellular responses to platinuminduced DNA damage. Chem Rev 107: 1387-1407, 2007.

4 Jamieson ER and Lippard SJ: Structure, recognition, and processing of cisplatin-DNA adducts. Chem Rev 99: 2467-2498, 1999.

5 Wang D and Lippard SJ: Cellular processing of platinum anticancer drugs. Nat Rev Drug Discov 4: 307-320, 2005.

6 Weidmann AG and Barton JK: A monofunctional platinum complex coordinated to a rhodium metalloinsertor selectively binds mismatched DNA in the minor groove. Inorg Chem 54: 9626-9636, 2015.

7 Liu F, Suryadi J and Bierbach U: Cellular recognition and repair of monofunctional-intercalative platinum-DNA adducts. Chem Res Toxicol 28: 2170-2178, 2015.

8 Park GY, Wilson JJ, Song Y and Lippard SJ: Phenanthriplatin, a monofunctional DNA-binding platinum anticancer drug candidate with unusual potency and cellular activity profile. Proc Natl Acad Sci USA 109: 11987-11992, 2012

9 Riddell IA, Johnstone TC, Park GY and Lippard SJ: Nucleotide binding preference of the monofunctional platinum anticanceragent phenanthriplatin. Chemistry 22: 7574-7581, 2016.

10 Lovejoy KS, Todd RC, Zhang S, McCormick MS, D'Aquino JA, Reardon JT, Sancar A, Giacomini KM and Lippard SJ: cisDiammine(pyridine)chloroplatinum(II), a monofunctional platinum(II) antitumor agent: Uptake, structure, function, and prospects. Proc Natl Acad Sci USA 105: 8902-8907, 2008.

11 Kelland L: The resurgence of platinum-based cancer chemotherapy. Nat Rev Cancer 7: 573-584, 2007.

12 Cvitkovic E: Ongoing and unsaid on oxaliplatin: the hope. Br J Cancer 77(Suppl 4): 8-11, 1998.
13 Wu S, Wang X, He Y, Zhu Z, Zhu C and Guo Z: A monofunctional trinuclear platinum complex with steric hindrance demonstrates strong cytotoxicity against tumor cells. J Inorg Biochem 139: 77-84, 2014.

14 Shen DW, Pastan I and Gottesman MM: In situ hybridization analysis of acquisition and loss of the human multidrug-resistance gene. Cancer Res 48: 4334-4339, 1988.

15 Adachi S, Obaya AJ, Han Z, Ramos-Desimone N, Wyche JH and Sedivy JM: c-Myc is necessary for DNA damage-induced apoptosis in the $\mathrm{G}(2)$ phase of the cell cycle. Mol Cell Biol 21: 4929-4937, 2001.

16 Maeda S, Sugiura T, Saikawa Y, Kubota T, Otani Y, Kumai K and Kitajima M: Docetaxel enhances the cytotoxicity of cisplatin to gastric cancer cells by modification of intracellular platinum metabolism. Cancer Sci 95: 679-684, 2004.

17 Endo K, Maehara Y, Kusumoto T, Ichiyoshi Y, Kuwano M and Sugimachi K: Expression of multidrug-resistance-associated protein (MRP) and chemosensitivity in human gastric cancer. Int J Cancer 68: 372-377, 1996.

18 Tanaka M, Kataoka H, Yano S, Ohi H, Kawamoto K, Shibahara T, Mizoshita T, Mori Y, Tanida S, Kamiya T and Joh T: Anticancer effects of newly developed chemotherapeutic agent, glycoconjugated palladium (II) complex, against cisplatin-resistant gastric cancer cells. BMC Cancer 13: 237, 2013.

19 Li YT, Chua MJ, Kunnath AP and Chowdhury EH: Reversing multidrug resistance in breast cancer cells by silencing $\mathrm{ABC}$ transporter genes with nanoparticle-facilitated delivery of target siRNAs. Int J Nanomedicine 7: 2473-2481, 2012.

20 Cole SP, Sparks KE, Fraser K, Loe DW, Grant CE, Wilson GM and Deeley RG: Pharmacological characterization of multidrug resistant MRP-transfected human tumor cells. Cancer Res 54: 5902-5910, 1994.

21 Theou N, Gil S, Devocelle A, Julie C, Lavergne-Slove A, Beauchet A, Callard P, Farinotti R, Le Cesne A, Lemoine A, Faivre-Bonhomme L and Emile JF: Multidrug resistance proteins in gastrointestinal stromal tumors: Site-dependent expression and initial response to imatinib. Clin Cancer Res 11: 7593-7598, 2005.

22 Grim J, D'Amico A, Frizelle S, Zhou J, Kratzke RA and Curiel DT: Adenovirus-mediated delivery of p16 to p16-deficient human bladder cancer cells confers chemoresistance to cisplatin and paclitaxel. Clin Cancer Res 3: 2415-2423, 1997.

23 Rogakou EP, Pilch DR, Orr AH, Ivanova VS and Bonner WM: DNA double-stranded breaks induce histone H2AX phosphorylation on serine 139. J Biol Chem 273: 5858-5868, 1998.

24 Ewald B, Sampath D and Plunkett W: H2AX phosphorylation marks gemcitabine-induced stalled replication forks and their collapse upon S-phase checkpoint abrogation. Mol Cancer Ther 6: 1239-1248, 2007.

25 Banath JP and Olive PL: Expression of phosphorylated histone $\mathrm{H} 2 \mathrm{AX}$ as a surrogate of cell killing by drugs that create DNA double-strand breaks. Cancer Res 63: 4347-4350, 2003.

26 Sai S, Vares G, Kim EH, Karasawa K, Wang B, Nenoi M, Horimoto $\mathrm{Y}$ and Hayashi M: Carbon ion beam combined with cisplatin effectively disrupts triple negative breast cancer stemlike cells in vitro. Mol Cancer 14: 166, 2015.

27 Tompkins JD, Wu X and Her C: MutS homologue hMSH5: role in cisplatin-induced DNA damage response. Mol Cancer 11: 10, 2012.

Received August 16, 2016

Revised September 3, 2016

Accepted September 6, 2016 